\title{
The Positivity of Gravitational Energy and Global Supersymmetry
}

\author{
C. M. Hull \\ Department of Applied Mathematics and Theoretical Physics, Silver Street, Cambridge, CB3 9EW, \\ England
}

\begin{abstract}
The concept of gravitational energy and the proof of its positivity are reviewed. The relationship between Witten's proof of the positivity of mass and supergravity is explained with reference to the group of global supersymmetries of a spacetime. A formula for the mass is given, in terms of the change of the supercharge under global supersymmetry, which has a simple positivity theorem and which reduces to Witten's expression. An interpretation of Witten's constraint on the spinors used in his proof is given.
\end{abstract}

\section{Introduction}

In special relativistic theories there is a conserved total 4-momentum and angular momentum associated with the translational and rotational invariance of flat space [1]. These first integrals of motion give important constraints on the dynamics, independent of the details of the mechanisms involved.

In general relativity, on the other hand, space-time is curved and in general has no global group of isometries. If the space-time has suitable asymptotic behaviour, however, one can define conserved charges associated with the generators of the group of motions of the asymptotic region. The case most often considered is that of a space whose metric tends asymptotically to that of Minkowski space, de Sitter space or anti-de Sitter space with the Poincaré group or the (anti-) de Sitter group as the asymptotic group. In particular, the energy is the charge associated with time translations.

Gravitational potential energy is negative since gravity is attractive. In Newtonian theory, the potential is unbounded below so that the energy of a gravitating system can be made arbitrarily negative by reducing its size, while in Einstein's theory the potential is even more negative. The energy of the gravitational field is non-local, since the Equivalence Principle implies that the strictly local effects of gravity can be removed by choosing a local inertial frame. The total energy receives positive contributions from matter and negative contributions from gravitational potential energy and so could, in principle, be of either sign. 
There are two types of mass that can be assigned to an asymptotically flat (AF) space which tends at infinity to Minkowski space giving the total mass in different three-surfaces. The ADM mass [2] is found by integrating an appropriate density over a space-like three-surface and gives the total mass of a space-time, including that of radiation, and is conserved. The Bondi mass [3] is associated with an asymptotically null three-surface and gives the mass not yet radiated away by a certain retarded time. The Bondi mass decreases with retarded time and cannot exceed the ADM mass.

Asymptotically anti-de Sitter (AAdS) spaces, however, only have one type of mass which was given by Abbott and Deser [4]. Since these spaces are not globally hyperbolic, information can both enter and leave at infinity and so the mass can either increase or decrease, only being strictly conserved if appropriate boundary conditions are imposed at infinity $[5,22]$.

An important recent development in gravity theory has been the establishment of various positive mass theorems. These prove that the total mass is non-negative, with a unique zero-energy configuration with appropriate boundary conditions, provided that the dominant energy condition holds, that is

$$
T_{\mu \nu} U^{\mu} V^{\nu} \geqq 0
$$

for all non-space-like vectors, $U^{\mu}, V^{\nu}$, where $T_{\mu \nu}$ is the stress energy tensor. Such theorems have been proved for AF spaces for both the ADM mass [6] and the Bondi mass [7], with Minkowski space as the unique zero energy state, and for AAdS spaces for the Abbott-Deser mass, with anti-de Sitter space as the unique AAdS zero energy configuration $[5,8]$.

The positivity of the Bondi and Abbott-Deser masses implies that gravitational energy can never become negative for AF and AAdS spaces, so that a system can never radiate away more energy than was originally present. It is believed that the reason the energy cannot be made arbitrarily negative by shrinking a system, as it could in Newtonian theory, is that if a system collapses beyond a certain size, a horizon forms, giving rise to a black hole which would appear to have positive mass to an observer outside the horizon. Regions of large negative energy and possible singularities would thus be hidden inside an horizon.

These theorems also imply the stability of the zero energy configuration to any decay process which preserves the boundary conditions, since there is no state of equal or smaller energy to which it can decay. [Note that both hot flat space [9] and the Kaluza-Klein vacuum [10] are unstable.]

The positive mass theorems can be generalised to hold for extended, gauged supergravity theories [5]. These theories have scalars with a potential that is unbounded below and so the scalar sector on its own would be pathological. However, the coupling to gravity stabilises the theory, with appropriate AAdS configurations having positive energy, and anti-de Sitter space can be a stable state in these models. Such classically and semi-classically stable ground states provide possible ground states about which one can attempt to construct a quantum theory.

Witten has given a simple proof of the positive energy theorem for AF spaces [11] which makes essential use of spinors and which was motivated by a formal 
argument $[12,13]$ based on the supersymmetry algebra which expressed the mass as a sum of squares.

It was by no means clear, however, why Witten's remarkably simple spinorial expression should give the ADM mass and how Witten's proof and the algebraic one are related. The purpose of this paper is to investigate these points. It will be seen that the mass of a system is given by the change of the supercharge under a global supersymmetry transformation, and the global algebra can be interpreted in this way. (In the Hamiltonian approach to supergravity, the Hamiltonian is given by the Dirac bracket of the supercharge with itself [14].) On the elimination of unphysical degrees of freedom this expression becomes a sum of squares and positivity of the mass follows.

The paper is organised as follows. In Sect. 2 , the proof of the positivity theorem is reviewed, in Sect. 3, the conserved charges are discussed and in Sect. 4, the algebraic proof is given. In Sect. 5, the algebra of global supersymmetries is investigated and a spinorial expression for the mass is derived. In Sect. 6, the positivity of this expression is shown, while in Sect. 7, the condition that Witten imposes on the spinors is interpreted. Section 8 is a conclusion.

\section{The Positive Mass Theorem}

Nester's formulation [15] of Witten's proof is fully covariant and avoids technical difficulties involved in taking the three-dimensional truncation of the four dimensional divergence theorem. It is also readily extended to the cases of the Bondi mass [16] and to AAdS space-times [5]. It will be assumed that the space-time is topologically trivial, with no horizons - this condition will later be relaxed. Consider the antisymmetric tensor ${ }^{1}$

$$
E^{\mu v}=\varepsilon^{\mu \nu \rho \sigma}\left(\bar{\varepsilon} \gamma^{5} \gamma_{\rho} \nabla_{\sigma} \varepsilon-\nabla_{\sigma} \bar{\varepsilon} \gamma^{5} \gamma_{\rho} \varepsilon\right)
$$

where $\varepsilon^{\alpha}$ is some commuting Dirac four-component spinor tending asymptotically to some constant spinor $\varepsilon_{0}$.

$$
\varepsilon \rightarrow \varepsilon_{0}+O\left(r^{-1}\right)
$$

The total ADM four-momentum $P_{\mu}$ of an AF space-time is given by [15]

$$
P_{\mu} \bar{\varepsilon}_{0} \gamma^{\mu} \varepsilon_{0}=\int_{S} \frac{1}{2} E^{\mu \nu} d \Sigma_{\mu \nu}
$$

where the surface integral is over the two-sphere at spatial infinity $S$ and the measure is given by

$$
\begin{gathered}
d \Sigma_{\mu \nu}=\frac{1}{2} \varepsilon_{\mu v \rho \sigma} d x^{\rho}{ }_{\Lambda} d x^{\sigma}, \\
d \Sigma_{\mu}=\frac{1}{6} \varepsilon_{\mu v \rho \sigma} d x^{v} d x^{\rho}{ }_{\Lambda} d x^{\sigma} .
\end{gathered}
$$

1 The metric signature is $(+---)$. Greek indices $\mu, v, \rho, \ldots=0,1,2,3$ refer to space-time components, $\alpha, \beta, \gamma, \ldots$ refer to spinor components, and Latin indices $a, b, c, \ldots$ refer to frame components,

$$
\left\{\gamma^{\mu}, \gamma^{\nu}\right\}=2 g^{\mu \nu}, \quad \gamma^{5}=\gamma^{0} \gamma^{1} \gamma^{2} \gamma^{3}, \quad \varepsilon_{0123}=e=\sqrt{ }-g, \quad \sigma_{a b}=\frac{1}{4}\left[\gamma_{a}, \gamma_{b}\right], \quad 8 \pi G=1
$$


Note that this includes a factor of the vierbein determinant since

$$
\varepsilon_{0123}=e=\sqrt{ }-g .
$$

Using the divergence theorem, (2.3) becomes a volume integral over a threesurface $\Sigma$ with boundary $S$,

$$
\begin{aligned}
P^{\mu} \bar{\varepsilon}_{0} \gamma^{\mu} \varepsilon_{0}= & \int_{\Sigma} \nabla_{v} E^{\mu \nu} d \Sigma_{\mu}=\int_{\Sigma} \varepsilon^{\mu v \rho \sigma}\left[\bar{\varepsilon} \gamma^{5} \gamma_{\rho} \nabla_{[v} \nabla_{\sigma]} \varepsilon+\nabla_{\nu} \bar{\varepsilon} \gamma^{5} \gamma_{\rho} \nabla_{\sigma} \varepsilon\right] d \Sigma_{\mu} \\
& +(\text { complex conjugate }) \\
= & \int_{\Sigma} G_{\mu \nu} \bar{\varepsilon} \gamma^{\mu} \varepsilon d \Sigma^{v}+\int_{\Sigma} \nabla_{\mu} \bar{\varepsilon}\left(\gamma^{\nu} \sigma^{\mu \rho}+\sigma^{\mu \rho} \gamma^{\nu}\right) \nabla_{\rho} \varepsilon d \Sigma_{\nu},
\end{aligned}
$$

where the Ricci identity has been used

$$
\left[\nabla_{v}, \nabla_{\sigma}\right] \varepsilon=\frac{1}{2} R_{v \sigma a b} \sigma^{a b} \varepsilon
$$

along with the identities

$$
\begin{aligned}
\gamma^{\mu} \sigma^{v \rho}+\sigma^{v \rho} \gamma^{\mu} & =\varepsilon^{\mu v \rho \sigma} \gamma_{\sigma} \gamma^{5}, \\
\frac{1}{4} \varepsilon^{\mu \nu \rho \sigma} R_{\rho \sigma}{ }^{a b} \varepsilon_{a b c d} & =* R^{* \mu v}{ }_{c d}, \\
e_{\mu}^{a *} R^{* \mu \nu}{ }_{a b} & =G_{b}^{v}{ }_{b} .
\end{aligned}
$$

where $G_{b}^{v}$ is the Einstein tensor.

As a result of the field equations

$$
G_{\mu \nu}=T_{\mu \nu},
$$

the first term in (2.7) is non-negative if $T_{\mu \nu}$ satisfies the dominant energy condition (1.1) as $\bar{\varepsilon} \gamma^{v} \varepsilon$ is non-space-like for Dirac spinors. Choosing co-ordinates so that the normal to $\Sigma$ is in the $x^{0}$ direction, the integrand in the second term in (2.7) becomes, with spatial indices $i, j=1,2,3$,

$$
2 \nabla_{i} \bar{\varepsilon} \gamma^{0} \sigma^{i j} \nabla_{j} \varepsilon=2\left(\nabla_{i} \varepsilon\right)^{\dagger} \sigma^{i j} \nabla_{j} \varepsilon=2\left|\nabla_{j} \varepsilon\right|^{2}-2\left|\sum_{j=1}^{3} \gamma^{j} \nabla_{j} \varepsilon\right|^{2} .
$$

This can be made positive by choosing $\varepsilon$ to satisfy the "Witten condition"

$$
\sum_{i=1}^{3} \gamma^{i} \nabla_{i} \varepsilon=0
$$

It has been shown that this can always be done [17], that is, that the elliptic differential equation (2.14) can always be solved, subject to the boundary conditions (2.2). Then, by (2.7), the energy integral is non-negative.

$$
\bar{\varepsilon}_{0} \gamma^{\mu} \varepsilon_{0} P_{\mu} \geqq 0,
$$

This must hold for any choice of constant spinor $\varepsilon_{0}$, and so $P_{\mu}$ is non-space-like and the energy of the space-time, $P^{0}$, is non-negative.

Further, $P^{\mu}$ is zero if and only if both

$$
T_{\mu \nu}=0
$$


and

$$
\nabla_{j} \varepsilon=0 .
$$

Equation (2.16) must hold for projections on to all possible surfaces $\Sigma$ with boundary $S$ and this is only possible [11] if

$$
\nabla_{\mu} \varepsilon=0 \text {. }
$$

The integrability condition for (2.17) for AF spaces is that the Riemann curvature vanishes and so the space-time must be flat.

If the three-surface $\Sigma$ is chosen to be asymptotically null, so that the two-surface $S$ is at null infinity, Eqs. (2.3) and (2.6) give the Bondi mass and the positivity follows in a similar manner [16]. It will be seen later that the Abbott-Deser mass is given by replacing the covariant derivative in $(2.1)$ by an "improved" derivative, given by (3.14), and again the proof proceeds as before $[5,11]$.

\section{Conserved Charges}

If a vector density $J^{\mu}$ satisfies $\partial_{\mu} J^{\mu}=0$, then $\int d^{3} x J^{0}$ is a charge which will be conserved if appropriate boundary conditions are imposed. For AF spaces the charges are conserved if the integral is over a space-like three-surface but not for asymptotically null hypersurfaces. In the AAdS case, by choosing the fields to satisfy certain boundary conditions [22] the integrals over space-like surfaces become conserved. In this paper, asymptotically null hypersurfaces will not be considered, although it seems that a similar analysis to that presented here should go through. The charges here discussed are associated with space-like surfaces and for AAdS spaces need not be conserved. In this section, following [4], charges will be constructed corresponding to the symmetries of the asymptotic region.

Suppose the metric $g_{\mu \nu}$ tends to a "background" metric $\bar{g}_{\mu \nu}$ at large distance

$$
g_{\mu \nu}=\bar{g}_{\mu \nu}+h_{\mu \nu},
$$

where $\bar{g}_{\mu \nu}$ is a solution of the Einstein equations $\left(\bar{G}_{\mu \nu}\right.$ is the Einstein tensor for $\left.\bar{g}_{\mu \nu}\right)$

$$
\bar{G}_{\mu \nu}+\Lambda \bar{g}_{\mu \nu}=0 \text {. }
$$

Of particular interest will be the spaces of constant curvature, since they have maximal symmetry. In flat space, or anti-de Sitter space with the metric in the globally static form [5], an appropriate asymptotic condition is

$$
h_{\mu \nu}=0\left(r^{-1}\right) \text { as } r \rightarrow \infty .
$$

The energy momentum tensor of the gravitational field $t_{\mu \nu}$ is defined by

$$
G^{\mu v}=\bar{G}^{\mu v}+G_{L}^{\mu \nu}-t^{\mu \nu},
$$

where $G_{L}^{\mu \nu}$ is linear in $h_{\mu \nu}$ and $t_{\mu \nu}$ consists of terms of second order and higher in $h_{\mu \nu}$. All operations such as raising and lowering indices and covariant differentiation are with respect to $\bar{g}_{\mu \nu}$ and will be until further notice. The linearised field equation is then

$$
G_{L}^{\mu \nu}+\Lambda h^{\mu v}=T^{\mu v}+t^{\mu v}=\Theta^{\mu \nu}
$$


The background Bianchi identity implies

$$
\bar{\nabla}_{\mu} \Theta^{\mu v}=0,
$$

where $\bar{\nabla}_{\mu}$ is the covariant derivative with respect to $\bar{g}_{\mu \nu}$. Let $k_{\mu}^{A}(A, B=1,2, \ldots)$ be the Killing vectors of the background metric $\bar{g}_{\mu v}$

$$
\bar{\nabla}_{\mu} k_{v}^{A}+\bar{\nabla}_{v} k_{\mu}^{A}=0
$$

so that the symmetries of the background metric correspond to Lie dragging along the integral curves of the Killing vector field. Then

$$
J_{\mu}^{A}=\bar{e} \Theta_{\mu \nu} k^{\nu A}
$$

is a conserved vector density where $\bar{e}=\sqrt{ }-\bar{g}$ is the determinant of the background vierbein, since

$$
\partial_{\mu}\left(\bar{e} \theta^{\mu v} k_{v}^{A}\right)=\bar{e} \bar{\nabla}_{\mu}\left(\theta^{\mu v} k_{v}^{A}\right)=0
$$

as a result of (3.6) and (3.7). Then

$$
K\left(k^{A}\right)=K^{A}=\int_{\Sigma} d^{3} x \bar{e} \theta^{\mu 0} k_{\mu}^{A}=\int_{\Sigma} \theta^{\mu v} k_{\mu}^{A} d \Sigma_{v}
$$

gives a set of conserved charges, one corresponding to each Killing vector field $k_{\mu}^{A}$. If $k_{\mu}^{A}$ is time-like, $K^{A}$ gives the energy relative to the zero-energy background state.

The conserved charges resulting from invariances of the background under supersymmetry transformations can be obtained in a similar way. In analogy with Killing vectors, Killing spinors [4] can be defined as spinor fields $\alpha$ generating local supersymmetries $\delta_{Q}(\alpha)$ leaving a configuration invariant. The infinitesimal supersymmetry variation of the gravitino field is given by

$$
\delta_{Q}(\alpha) \psi_{\mu}=\hat{\nabla}_{\mu} \alpha,
$$

which must vanish if $\alpha$ is to be a Killing spinor, where $\hat{\nabla}$ is some differential operator. For $N=1$ supergravity [18]

$$
\hat{\nabla}_{\mu} \alpha=\nabla_{\mu} \alpha,
$$

where $\nabla$ is the gravitational covariant derivative with torsion [18]

$$
T_{\mu \nu}^{a}=-\frac{1}{4} \bar{\psi}_{\mu} \gamma^{a} \psi_{\nu}
$$

and for $N=1$ supergravity with a cosmological term $\Lambda<0$, [19]

$$
\hat{\nabla}_{\mu}=\nabla_{\mu}+i \sqrt{ }(-\Lambda / 12) \gamma_{\mu} .
$$

In the $N=1$ theories, the gravitino field and the spinors $\alpha$ satisfy a Majorana (reality) condition.

Of particular interest are purely bosonic backgrounds with $\psi_{\mu}=0$, and hence vanishing torsion, so that $\nabla$ reduces to the usual gravitational covariant derivative. The supersymmetry variations of the bosonic fields are proportional to the fermionic fields and so vanish. From (3.11), a necessary condition for a spinor $\alpha$ to be a Killing spinor for a bosonic background is

$$
\tilde{\nabla}_{\mu} \alpha=0,
$$


where $\tilde{\nabla}_{\mu}$ is the $\hat{\nabla}$ for the background $\bar{g}_{\mu \nu}$. For $N=1$ supergravity, the Killing spinors are covariantly constant spinors. The integrability condition for (3.15) is

$$
\tilde{\nabla}_{[\mu} \tilde{\nabla}_{v]} \alpha=0 \text {. }
$$

The super curvature $\hat{\nabla}_{[\mu} \hat{\nabla}_{\nu]}$ vanishes if $\bar{g}_{\mu \nu}$ is the metric for a space of constant non-positive curvature, with $\tilde{\nabla}_{\mu}$ given by (3.12) or (3.14), with $\Lambda$ being the appropriate cosmological constant. There would then be a four-space of linearly independent solutions to (3.15).

The anti-commuting Killing spinor $\alpha$ can then be expressed as a linear combination of terms of the form $\rho^{m} \alpha^{m}(x), m=1,2, \ldots, \rho^{m} \rho^{n}=-\rho^{n} \rho^{m}$, where $\rho^{m}$ is an odd element of the Grassman algebra and the $\alpha^{m}$ form a set of linearly independent commuting spinor fields, each of which satisfies (3.15).

The Rarita-Schwinger equation can be split into linear and non-linear parts as follows

where

$$
\varepsilon^{\mu \nu \rho \sigma} \gamma^{5} \bar{\gamma}_{\nu} \tilde{\nabla}_{\rho} \psi_{\sigma}=R^{\mu}
$$

$$
\bar{\gamma}_{v}=\bar{e}_{v}^{a} \gamma_{a}
$$

and $R^{\mu}$ consists of non-linear and source terms. Here $\hat{\nabla}_{[\rho} \psi_{\sigma]}$ is a super-covariant curl [20]. Then $R^{\mu}$ satisfies

$$
\tilde{\nabla}_{\mu} R^{\mu}=0
$$

from (3.17). Then for a commuting Killing spinor $\alpha^{m}$

$$
\begin{aligned}
\bar{e} \bar{\alpha}^{m} R^{\mu} & =\bar{e} \bar{\alpha}^{m} \varepsilon^{\mu \nu \rho \sigma} \gamma^{5} \bar{\gamma}_{\nu} \tilde{\nabla}_{\rho} \psi_{\sigma}=e \bar{\nabla}_{\rho}\left(\bar{\alpha}^{m} \varepsilon^{\mu \nu \rho \sigma} \gamma_{5} \bar{\gamma}_{v} \psi_{\sigma}\right) \\
& =\partial_{\rho}\left(\bar{e} \alpha^{m} \varepsilon^{\mu \nu \rho \sigma} \gamma_{5} \bar{\gamma}_{\nu} \psi_{\sigma}\right) .
\end{aligned}
$$

Then from (3.20)

so that

$$
\partial_{\mu}\left(\bar{e} \bar{\alpha}^{m} R^{\mu}\right)=0
$$

$$
Q^{m}=\int_{\Sigma} d^{3} x \bar{e} \bar{\alpha}^{m} R^{0}=\int_{\Sigma} \bar{\alpha}^{m} \varepsilon^{\mu \nu \rho \sigma} \bar{\gamma}_{\nu} \tilde{\nabla}_{\rho} \psi_{\sigma} d \Sigma_{\mu}
$$

gives a set of conserved anti-commuting supercharges, one corresponding to each Killing spinor $\alpha^{m}$. The supercharges can be expressed as surface integrals using (3.20) over $S=\partial \Sigma$,

$$
Q^{m}=\int_{S} \frac{1}{2} \bar{\alpha}^{m} \varepsilon^{\mu \nu \rho \sigma} \gamma_{5} \bar{\gamma}_{\nu} \psi_{\sigma} \Sigma_{\mu \rho}
$$

The charges $K^{A}$ given by (3.10) can also be re-written as surface integrals $[1,4]$. Let $\Gamma_{b}^{a}=\Gamma_{\mu b}^{a} d x^{\mu}$ be the connection one forms for $h_{\mu v}$, so that

$$
\Gamma_{b}^{a}=\Gamma_{b}^{a}\left(h_{\mu v}\right)=\Gamma_{b}^{a}\left(g_{\mu \nu}\right)-\Gamma_{b}^{a}\left(\bar{g}_{\mu \nu}\right)
$$

and so is a tensor. Then [15], for $\Lambda=0$,

$$
K^{A}=\frac{1}{4} \int_{S} \delta_{\mu \nu \rho}^{\sigma \tau \lambda} \Gamma_{b}^{v a} k^{A \rho} \bar{e}_{a}^{\mu} \bar{e}_{\lambda}^{b} d \Sigma_{\sigma \tau}
$$


which gives (3.10) on using the divergence theorem where

$$
\delta_{\mu \nu \rho}^{\sigma \tau \lambda}=\varepsilon^{\sigma \tau \lambda \kappa} \varepsilon_{\mu \nu \rho \kappa} .
$$

Defining the forms

$$
e^{a}=e_{\mu}^{a} d x^{\mu}, \quad k^{A}=k_{\mu}^{A} d x^{\mu}, \quad R_{a b}=\frac{1}{2} R_{\mu v a b} d x_{\Lambda}^{\mu} d x^{\nu},
$$

the conserved charges can be re-expressed as

$$
\begin{aligned}
& K^{A}=\int_{\Sigma} R_{a b \Lambda}^{L} *\left(k^{A}{ }_{\Lambda} \bar{e}^{a}{ }_{\Lambda} \bar{e}^{b}\right), \\
& K^{A}=\int_{S} \Gamma_{a b \Lambda} *\left(k^{A}{ }_{\Lambda} \bar{e}^{a}{ }_{\Lambda} \bar{e}^{b}\right) .
\end{aligned}
$$

Expressions for $\Lambda \neq 0$ will be given later.

The surface integrals (3.23) and (3.25) remain unchanged if the metric $\bar{g}_{\mu \nu}$ is replaced by $g_{\mu \nu}$ in these expressions and all contractions are performed with $g_{\mu \nu}$. There would also be no change if the Killing vectors and spinors were replaced by vectors and spinors that tend asymptotically to Killing vectors and spinors. For AF spaces, asymptotic Killing spinors would be constant spinors plus terms of order $1 / r$. With these replacements, there would be extra terms if the surface integrals were reexpressed as volume integrals, resulting from the derivatives of asymptotic Killing quantities.

In the case of space-times with non-simple topology, as is the case when horizons are present, the background splitting

$$
g_{\mu \nu}=\bar{g}_{\mu \nu}+h_{\mu \nu}
$$

becomes problematic, but it is still useful in the asymptotic region where (3.30) still holds with $h_{\mu \nu}$ small. The volume integral expressions for the charges, (3.10) and (3.22) are then not well-defined and the charges should be defined by their surface integral expressions in terms of asymptotic Killing vectors and spinors.

On using the divergence theorem these expressions give a volume integral together with surface integrals over internal boundaries. Since it is only the asymptotic behaviour of the asymptotic Killing spinors and vectors that is important, they can be chosen so that the surface integrals over the internal boundaries vanish.

In the following sections it will prove useful to first consider simple topology and use the volume integrals (3.10) and (3.22) and then relax the assumption and work with the more general surface expressions.

\section{The Global Super-Algebra}

The quantum operators generating the global symmetries and supersymmetries corresponding to the background Killing vectors and spinors satisfy a global supersymmetry algebra.

The action of these symmetries on fields will be generated by the graded commutators of the fields with the surface integrals given in Sect. 3 only if all local gauge freedom is eliminated from the fields present, [12]. Only then will the 
variation of a field at infinity determine the transformation everywhere. In the AF case one obtains the super-Poincaré algebra consisting of the Poincare algebra satisfied by the set of charges $K^{A}$ consisting of the global 4-momentum $P^{M}$ and the global angular momentum $J^{M N}=-J^{N M}, M, N=0,1,2,3$, together with the relations

$$
\begin{gathered}
{\left[P^{M}, Q^{m}\right]=0,} \\
{\left[J^{M N}, Q^{m}\right]=\hbar \sigma_{n}^{M N m} Q^{n},} \\
\left\{Q^{m}, \bar{Q}^{n}\right\}=\hbar \gamma_{M}^{m n} P^{M} .
\end{gathered}
$$

In the AAdS case, the appropriate relations are these of the graded anti-de Sitter algebra, $\mathrm{OSp}(1 / 4)$, with bosonic generators $J_{M N}=-J_{N M}$ and $J_{M 4}, M, N=0, \ldots, 3$, satisfying the $0(3.2)$ algebra. Relation (4.3) is replaced by

$$
\left\{Q^{m}, \bar{Q}^{n}\right\}=\hbar \gamma_{M}^{m n} J^{M 4}+i \hbar \sigma_{M N}^{m n} J^{M N} .
$$

Since the fields, $e_{\mu}^{a}, \psi_{\mu}$ are constrained by the gauge conditions imposed, their commutation relations are modified and the brackets occurring in the algebras are the quantum analogues of Dirac brackets [21].

The relations (4.3), (4.4) lead to a simple algebraic proof of the positivity of energy in quantum supergravity [12]. Taking the expectation of (4.3) for a physical state $|s\rangle$ and multiplying by $\gamma^{0}$ yields

$$
\hbar^{-1}\left\langle s\left|\left\{Q^{m}, Q^{n \dagger}\right\}\right| s\right\rangle=\left(\gamma^{M} \gamma^{0}\right)^{m n}\left\langle s\left|P_{M}\right| s\right\rangle .
$$

The manifest positivity of the left-hand side implies the positivity of the eigenvalues of $\gamma^{0} \gamma^{A} P_{A}$ which are $P^{0} \pm|P|$. The positivity of the expectation value of $P^{0}$ can also be obtained by taking the trace of (4.5). The positivity of the energy $J^{04}$ for AAdS spaces can be shown in the same way using $(4.4),[4,22]$. This suggests the positivity of energy in classical Einstein gravity [13], since one may consider the expectation value (4.5) for purely bosonic states $|s\rangle$, with vanishing gravitino field and hence zero supercharge, and then take the limit $h \rightarrow 0$. This is not rigorous, however, since it requires the existence of a consistent, supersymmetric quantum theory of gravity.

Witten's proof resulted from an attempt to find a rigorous classical version of this formal quantum argument. Classical supergravity can be considered as a field theory enjoying local supersymmetry, with fields taking values in a Grassman algebra and the graded commutation relations of the quantum theory replaced by graded Poisson brackets without factors of $h$.

In the Hamiltonian formulation [23], constraints are imposed and the Poisson brackets become Dirac brackets. The surface integrals then again satisfy a global supersymmetry algebra, [14]. Witten's argument can be understood as arising from this interpretation of the algebra (4.3). (This has also been noticed in [24].) However, it is much more illuminating to consider the graded Lie algebra of global symmetries. Since these symmetry transformations can be represented by the Dirac bracket, or corresponding quantum commutator, of the charges with the fields, this approach will imply the results of the interpretation of the super-algebra in terms of both classical and quantum Dirac brackets. 


\section{Global Symmetries}

The commutator of two vector fields $U, V$ is given by the Lie derivative $£ V$,

$$
[U, V]=\underset{U}{£} V .
$$

The commutator of two Killing vector fields of $\bar{g}_{\mu \nu}$ will again be a Killing vector field

$$
\left[k_{A}, k_{B}\right]=C_{A B}^{C} k_{C}
$$

for some constants $C_{A B}^{C}$. Consider the "conjugate" operators $\widetilde{K}^{A}$ which generate infinitesimal isometries by Lie dragging along the integral curves of the vector fields $k^{A}$. Infinitesimal general co-ordinate transformations depending on some infinitesimal parameter $t$ are then given by

$$
t \tilde{K}^{A}=\delta_{G C T}\left(k_{A} t\right)=t £_{k^{A}},
$$

and finite group elements are obtained by exponentiation. Since Lie derivatives satisfy the relation, for any vector fields $U, V$,

$$
\underset{U V}{f} \underset{V U}{f} \underset{[U, V]}{£}=\underset{[0}{£}
$$

the symmetry generators satisfy the algebra

$$
\left[\tilde{K}_{A}, \tilde{K}_{B}\right]=C_{A B}^{C} \tilde{K}_{C}
$$

with the same structure constants $C_{A B}^{C}$ as in (5.2). For AF spaces this will be the Poincare algebra and for AAdS spaces it will be the anti-de Sitter algebra (the algebra of $0(3,2))$.

The background space-time will transform as a massless representation of this symmetry group, while the asymptotic region of the full space-time will in general transform according to a massive representation.

One can also consider the transformation of the charges $K_{B}$ under the global symmetries $\tilde{K}_{\boldsymbol{A}}$

$$
\left[\tilde{K}_{A}, K_{B}\right]=\delta_{G C T}\left(k_{A}\right) \int \theta_{\mu \nu} k_{B}^{\mu} d \Sigma^{v}=C_{A B}^{c} K_{C} .
$$

Thus, as one would expect, the charges $K_{B}$ are just "rotated" into each other by the global symmetries. This analysis extends readily to classical supergravity. The commutator of two local supersymmetry transformations $\delta_{Q}\left(\varepsilon_{1}\right)$ and $\delta_{Q}\left(\varepsilon_{2}\right)$ is given, if acting on on-shell states, by

$$
\left[\delta_{Q}\left(\varepsilon_{1}\right), \delta_{Q}\left(\varepsilon_{2}\right)\right]=\delta_{G C T}\left(\xi^{\mu}\right)+\delta_{Q}\left(-\xi^{\mu} \psi_{\mu}\right)+\delta_{L}\left(\xi^{\mu} \Gamma_{\mu}^{a b}\right), \quad \xi^{\mu}=\frac{1}{2} \bar{\varepsilon}_{2} \gamma^{\mu} \varepsilon_{1},
$$

where $\Gamma_{\mu}^{a b}$ is the spin connection and $\delta_{L}$ represents a local Lorentz rotation. If $\varepsilon_{1}$ and $\varepsilon_{2}$ are Killing spinors, the right-hand side must be a pure gauge transformation and so $\xi^{\mu}$ must be a Killing vector and $\xi^{\mu} \psi_{\mu}$ must be a Killing spinor.

This implies a relation between the commuting Killing spinors $\alpha_{m}$ and the Killing vectors $k_{A}$

$$
\bar{\alpha}_{m} \gamma^{\mu} \alpha_{n}=f_{m n}^{A} k_{A}^{\mu}
$$

for some constants $f_{m n}^{A}$. The basis of Killing spinors $\alpha^{m}$ can be chosen [4,22] so that 
for flat space

$$
\bar{\alpha}_{m} \gamma^{\mu} \alpha_{n}=\gamma_{n m}^{M} k_{M}^{\mu}
$$

and for AdS space

$$
\bar{\alpha}_{m} \gamma^{\mu} \alpha_{n}=\gamma_{n m}^{M} k_{M}+i \sigma_{n m}^{M N} k_{M N} .
$$

Here $M, N=0, \ldots, 3$ and the $k_{M}$ are the four Killing vectors generating global translations. The charges corresponding to these Killing vectors are

$$
K\left(k^{M}\right)=P^{M}, \quad\left[\text { or } \quad K\left(k^{M}\right)=J^{M 4} \text { for AdS case }\right], \quad K\left(k^{M N}\right)=J^{M N} .
$$

The $\gamma_{m n}^{M}$ are the usual gamma matrices satisfying $\left\{\gamma^{M}, \gamma^{N}\right\}=2 \eta^{M N}$. Consider the generators of infinitesimal global supersymmetries generated by Killing spinors

$$
\tilde{Q}_{m}=\delta_{\bar{Q}}\left(\bar{\alpha}_{m}\right), \quad \tilde{Q}_{m}=\delta_{Q}\left(\alpha^{m}\right) .
$$

These will satisfy the algebra

$$
\left\{\widetilde{Q}_{m}, \tilde{\bar{Q}}_{n}\right\}=f_{m n}^{M} \widetilde{K}_{M}
$$

with the same structure constants $f_{m n}^{M}$ as in (5.7), thus giving the same algebra as (4.3) or (4.4) in the appropriate cases.

In analogy with (5.5) one might expect the transformation of the supercharges $Q_{n}$ under the action of the global supersymmetries $\widetilde{Q}_{m}$ to be given by

$$
\left\{\tilde{Q}_{m}, Q_{n}\right\}=\delta_{Q}\left(\alpha^{m}\right) Q_{n}=f_{m n}^{A} K_{A}
$$

This will be shown to be the case and (5.13) can be used to express the mass in classical supergravity in terms of the change of the supercharge under supersymmetry. This is defined even for systems with vanishing gravitino field, and hence vanishing supercharge, and in this limit the expression reduces to those of Witten and Nester for the mass in general relativity.

The supercharge is given by

$$
Q^{n}=\int_{\Sigma} \bar{\alpha}^{n} R^{\mu} d \Sigma_{\mu}
$$

The result follows essentially since, under the action of supersymmetry, fermionic field equations transform into bosonic field equations and in particular the linearized gravitino field equation $R^{\mu}$ transforms into the linearized Einstein field equation $G_{L}^{\mu \nu}+\Lambda h^{\mu \nu}$. In the AF case, factoring out Grassman parts,

$$
\delta_{Q}\left(\alpha^{m}\right) \psi_{\mu}=\nabla_{\mu} \alpha^{m}+O\left(\psi^{2}\right)
$$

where $\nabla_{\mu}$ is here the covariant derivative for the full metric $g_{\mu \nu}$ without torsion. The torsion (3.13) is of quadratic order in the gravitino field $\psi_{u}$, such terms being represented by $O\left(\psi^{2}\right)$. [Note that $\tilde{\nabla}_{\mu} \alpha^{m}=\bar{\nabla}_{\mu} \alpha^{m}=0$ but $\nabla_{\mu} \alpha^{m} \neq 0$.] Then

$$
\delta_{Q}\left(\alpha^{m}\right) R^{\mu}=\varepsilon^{\mu v \rho \sigma} \gamma^{5} \bar{\gamma}_{v} \bar{\nabla}_{\rho} \nabla_{\sigma} \alpha_{m}+O\left(\psi^{2}\right)=\varepsilon^{\mu \nu \rho \sigma} \bar{e}_{v}^{c} R_{\rho \sigma}^{L a b}\left(\gamma^{5} \gamma_{c} \sigma_{a b}\right) \alpha^{m}+O\left(\psi^{2}\right),
$$

where

$$
\bar{\nabla}_{[\rho} \nabla_{\sigma]} \alpha=\frac{1}{4} R_{\rho \sigma a b}^{L} \sigma^{a b} \alpha,
$$


and $R_{\rho \sigma a b}^{L}$ is the part of the curvature tensor linear in $h_{\mu v}$. Using

$$
\gamma_{c} \sigma_{a b}=\frac{1}{2}\left(\eta_{a c} \gamma_{b}-\eta_{b c} \gamma_{a}+\varepsilon_{a b c d} \gamma^{5} \gamma_{d}\right)
$$

Eqs. (2.10), (2.11) and the Bianchi identity $\varepsilon_{\mu \nu \rho \tau} R_{L}^{\mu \nu \rho \sigma}=0$, one obtains

$$
\delta_{Q}\left(\alpha^{m}\right) R^{\mu}={ }^{*} R_{L}{ }^{* \mu \nu}{ }_{c d} \bar{e}^{c}{ }_{v} \gamma^{d} \alpha^{m}+O\left(\psi^{2}\right)=G_{L d}^{\mu} \gamma^{d} \alpha_{m}+O\left(\psi^{2}\right) .
$$

Then the variation of the supercharge, regarded as an active transformation acting on the fields leaving the co-ordinate systems and Killing fields invariant, is

$$
\begin{aligned}
\delta_{Q}\left(\alpha^{m}\right) Q^{n} & =\int_{\Sigma} \bar{\alpha}^{n} \delta_{Q}\left(\alpha^{m}\right) R^{\mu} d \Sigma_{\mu}=\int_{\Sigma} \theta^{\mu v} \bar{\alpha}^{n} \gamma_{\mu} \alpha^{m} d \Sigma_{\nu}+O\left(\psi^{2}\right) \\
& =\gamma_{M}^{m n} P^{M}+O\left(\psi^{2}\right) .
\end{aligned}
$$

The variation of the vierbein gives rise to terms quadratic in the gravitino field.

If one instead considers the surface integral form for $Q^{n}(3.23)$.

$$
\delta_{Q}\left(\alpha^{m}\right) Q_{n}=\int_{S} \frac{1}{2} \varepsilon^{\mu \nu \rho \sigma}\left(\bar{\alpha}_{n} \gamma^{5} \bar{\gamma}_{\rho} \nabla_{\sigma} \alpha_{m}\right) d \Sigma_{\mu \nu}+O\left(\psi^{2}\right)
$$

using (5.18) and

$$
\nabla_{\sigma} \alpha^{m}=\bar{\nabla}_{\sigma} \alpha^{m}+\left(\Gamma_{\sigma}^{a b} \sigma_{a b}\right) \alpha^{m}=\left(\Gamma_{\sigma}^{a b} \sigma_{a b}\right) \alpha^{m}
$$

where $\Gamma_{\sigma}^{a b}$ is the connection for $h_{\mu \nu}$ given by (3.24), one obtains

$$
\delta_{Q}\left(\alpha^{m}\right) Q_{n}=\frac{1}{4} \int_{S} \delta_{\mu \nu \rho}^{\sigma \tau \lambda} \Gamma_{b}^{v a} \bar{e}_{a}^{\mu} \bar{e}_{\lambda}^{b} \bar{\alpha}^{n} \gamma^{\rho} \alpha^{m} d \Sigma_{\sigma \tau}+O\left(\psi^{2}\right)
$$

so by (3.25),

$$
\delta_{Q}\left(\alpha^{m}\right) Q_{n}=\gamma_{m n}^{M} P_{M}+O\left(\psi^{2}\right) .
$$

In fact (5.13) holds quite generally since it is a consequence of the fact that fermionic field equations transform into field equations and for systems with vanishing gravitino fields

$$
f_{m n}^{A} K_{A}=\int_{S} \frac{1}{2} \varepsilon^{\mu v \rho \sigma}\left(\bar{\alpha}_{n} \gamma^{5} \bar{\gamma}_{\rho} \hat{\nabla}_{\sigma} \alpha_{m}\right) d \Sigma_{\mu \nu},
$$

with $\hat{\nabla} \alpha$ as defined in (3.11). For AF and AAdS systems, the mass can be obtained by multiplying by $\gamma_{n m}^{0}$ and summing over $m$ and $n$. The expressions for the mass used in positivity theorems will derive from the simple expression (5.25) in terms of Killing spinors.

\section{Positivity}

The total mass given by (3.10) is not manifestly positive since $\theta^{00}$ can have either sign. It is therefore desirable to try to find expressions in terms of the stress-energy $T^{\mu \nu}$ which satisfies the dominant energy condition (1.1), or in other words to work with the full field equations rather than the linearized ones.

The values of the charges given by (5.25) remain unchanged if all contractions, derivatives etc., are performed with respect to the full metric $g_{\mu \nu}$ instead of $\bar{g}_{\mu \nu}$, since $h_{\mu \nu}$ has appropriate asymptotic behaviour. The Killing spinors can also be replaced 
with asymptotic Killing spinors, $\chi$,

$$
\chi(x)=\sum_{n} \chi_{m}(x) \alpha_{m}(x), \quad \chi_{m}(x) \rightarrow \chi_{m}^{0} \quad \text { as } \quad r \rightarrow \infty
$$

for some constant $\chi_{m}^{0}$. Then

$$
\left(\bar{\chi}_{0}^{n} f_{m n}^{A} \chi_{0}^{m}\right) K_{A}=\int_{S} \frac{1}{2} \varepsilon^{\mu \nu \rho \sigma} \bar{\chi} \gamma^{5} \gamma_{\rho} \hat{\nabla}_{\sigma} \chi d \Sigma_{\mu \nu},
$$

where here, and for the rest of the paper, all operations are with respect to the full metric. The simple expression (6.1) for the mass holds for arbitrary asymptotic Killing spinors $\chi$ and in the presence of a negative cosmological constant. Since for commuting Majorana spinors a Fiertz rearrangement gives

$$
\bar{\alpha}_{n} \gamma^{5} \gamma_{\rho} \hat{\nabla}_{\sigma} \alpha_{m}=-\left(\hat{\nabla}_{\sigma} \alpha_{m}\right) \gamma^{5} \gamma_{\rho} \alpha_{n},
$$

by taking complex valued functions $\chi_{m}(x)$ so that $\chi$ becomes a Dirac spinor, Nester's expression for the mass $(2.1),(2.3)$ is regained.

On using the divergence theorem, if the topology is trivial, (6.1) can be put into the form

$$
\left(\bar{\chi}_{0}^{n} f_{m n}^{A} \chi_{0}^{m}\right) K_{A}=\int_{\Sigma} \varepsilon^{\mu \nu \rho \sigma}\left[\bar{\chi} \gamma^{5} \gamma_{\rho} \hat{\nabla}_{\nu} \hat{\nabla}_{\sigma} \chi+\overline{\left(\hat{\nabla}_{\nu} \chi\right)} \gamma^{5} \gamma_{\rho} \hat{\nabla}_{\sigma} \chi\right] d \Sigma_{\mu}
$$

For AF and AAdS spaces, with $\hat{\nabla}$ given by (3.12) or (3.14) respectively, the first term on the right hand side of (6.2) yields, on using the field equations,

$$
\int_{\Sigma} T^{\mu \nu} \bar{\chi} \gamma_{\mu} x d \Sigma_{v}
$$

which is positive if the dominant energy condition (1.1) is assumed to hold. A result similar to (6.3) obtains, with some modification, for other supergravity models $[5,25]$ since it is again just a result of the fact that the supersymmetry variation of the Rarita-Schwinger equation gives the Einstein equation. The remaining term in (6.2) is positive, by the reasoning presented in Sect. 2, if the Witten condition

$$
\sum_{j=1}^{3} \gamma^{j} \hat{\nabla}_{j} \chi=0
$$

is imposed, as can always be done in the AF case [17] and is presumably also possible in the general case. Then $\bar{\chi}_{0}^{n} f_{m n}^{A} \chi_{0}^{m} K_{A}$ is positive for all spinors $\chi_{0}$ which implies the positivity of the mass for the choices of $f_{m n}^{A}$ of interest. On using the Witten condition (6.4), the expression (6.1) reduces to

$$
\left(\bar{\chi}_{0}^{n} f_{m n}^{A} \chi_{0}^{m}\right) K_{A}=\int_{S} \chi^{\dagger} \hat{\nabla}_{j} \chi d \Sigma^{o j}
$$

a generalization of the formula for the mass given by Witten [11], where the coordinates are chosen such that the surface of integration $S$ is the boundary of a threesurface $\Sigma$ given by $x^{0}=$ constant and $i=1,2,3$ labels the spatial components.

For the mass given by (6.1) to be zero it is necessary that $\chi$, which is an asymptotic 
Killing spinor of the background space, $\hat{\nabla} \chi \rightarrow 0$, also be an exact Killing spinor of the full space

$$
\hat{\nabla}_{\mu} \chi=0 \text {. }
$$

For $\hat{\nabla}$ as given in (3.12) or (3.14) this implies that $h_{\mu \nu}=0$ and the full space must be Minkowski space or anti-de Sitter space respectively and these are the unique zero energy AF or AAdS configurations.

If the topology is non-trivial, expression (6.2) will be modified by the addition of surface integrals over internal boundaries. It has been shown [8] that, if the internal boundaries form an apparent horizon, (the possibly disconnected outer boundary of closed trapped surfaces) the spinor $\chi$ can be chosen so that it satisfies the Witten condition (6.4) and such that the surface integral over the apparent horizon vanishes. If the space-time is regular outside the apparent horizon, the proof proceeds as before and the mass is positive.

\section{The Witten Condition}

At first sight it seems strange that in the classical proof the Witten condition (6.4) must be imposed, for there seems to be no analogue in the algebraic argument of Sect. 4. In fact such a condition is needed since $|s\rangle$ in (4.5) must be a physical state. It is necessary to impose gauge conditions and constraints on the fields to remove nonphysical, "longitudinal" degrees of freedom which may have negative norm. Then and only then does the space of states have positive definite norm. Since $Q$ is an Hermitian operator on that Hilbert space, it then follows that the mass of all physical states is positive.

To this end, it is appropriate to make a canonical decomposition or $3+1$ split [23] tailored to the three-surface $\Sigma$, the mass associated with which is being considered. The time-component of the gravitino field $\psi_{0}$ is then non-propagating and the dynamical part of the Rarita-Schwinger field is given by $\psi_{j}, j=1,2,3$, subject to a gauge choice such as

$$
\sum_{j=1}^{3} \gamma^{j} \psi_{j}=0 .
$$

The parameters $\varepsilon$ of local supersymmetry transformations must be restricted so as to maintain the constraints and gauge conditions. Condition (7.1) is preserved if

$$
\sum_{j=1}^{3} \gamma^{j} \widehat{\nabla} \varepsilon=0,
$$

which is precisely the Witten condition. Transformations given by Dirac brackets with the global charges will automatically preserve these conditions.

The mass of a source-free gravitational field is given by (6.1) in terms of the change of the supercharge $\chi_{0}^{m} Q_{m}$ by an arbitrary global supersymmetry $\delta_{Q}(\chi)$ with parameter $\chi$, where $\chi$ is an arbitrary asymptotic Killing spinor.

$$
\left(\bar{\chi}_{0}^{n} f_{m n}^{A} \chi_{0}^{m}\right) K_{A}=\int_{\Sigma}\left(\hat{\nabla}^{j} \chi\right)^{\dagger}\left(\hat{\nabla}_{j} \chi\right) e d^{3} x-\int_{\Sigma}\left(\gamma^{j} \hat{\nabla}_{j} \chi\right)^{\dagger}\left(\gamma^{j} \widehat{\nabla}_{j} \chi\right) e d^{3} x
$$


If the gauge condition (7.1) is imposed, $\chi$ must satisfy (7.2) so that (7.3) becomes a "sum of squares" and is positive. If, however, (7.1) is not imposed, the gravitino field transforms into a gravitational field composed of both positive-energy, physical parts and negative energy, unphysical parts. Since supersymmetry transforms physical states into physical states, the positive term on the right hand side of (7.3) gives the total energy of the physical part of the gravitational field, while the negative term gives the negative energy of the unphysical parts. The negative term thus gives a gauge-dependent contribution to the mass that can be set to zero by choosing a physical gauge for the gravitational field.

\section{Conclusion}

The mass of any classical supergravity configuration that tends asymptotically to a supersymmetric background space-time can be given relative to that of the background by the change of the supercharge under an infinitesimal global supersymmetry transformation. In particular this has been seen to be true for general relativistic systems which have vanishing gravitino field and hence supercharge. (A supersymmetric background is one that is invariant under global supersymmetry and so admits a Killing spinor). If all the non-physical degrees of freedom are eliminated, then this expression can be used to show the positivity of mass of systems consisting of Einstein gravity, coupled to any matter distribution satisfying the dominant energy condition (1.1) that is regular outside an apparent horizon and has appropriate asymptotic behaviour. For AF and AAdS spaces, the maximally supersymmetric space-time is the unique zero energy state. It is, of course, not possible to compare the energies of spaces with different asymptotic behaviour.

A key role in the analysis was played by the fact that the supersymmetry variation of the Rarita-Schwinger equation is proportional to the Einstein equation in $N=1$ supergravity (and similarly for the linearized field equations) which is a result of the supercurrent and the energy-momentum tensor lying in the same supermultiplet. Since the charges can be expressed in terms of the linearized field equations, the relation between mass and supercharge follows.

The proof of positivity cannot be extended to spaces with positive cosmological constant since the operator $\hat{\nabla}$ given by (3.14) with $\Lambda>0$ no longer has the necessary properties. This is related to the fact that the de Sitter group $0(4,1)$ cannot be graded.

The results discussed here can be extended in various ways. By including all the terms of quadratic order and higher in the gravitino field, a proof of the positivity of mass in classical supergra vity can presumably be obtained. In extended supergravity theories, a lower bound for the mass can be given in terms of the central charges. For example, in $N=2$ supergravity, which reduces to Einstein-Maxwell theory for purely bosonic configurations, the mass $M$ satisfies

$$
M-\left(Q^{2}+P^{2}\right)^{1 / 2} \geqq 0
$$

with equality if and only if the system is globally supersymmetric, where $Q$ is the total electric charge, $P$ is the total magnetic charge and it is assumed that the local mass and charge densities satisfy an inequality similar to (8.1) [26]. 
The inequality (7.1) is further modified if there is a non-zero NUT-charge or magnetic mass [25]. Finally, the methods discussed here extend to give the positivity of mass and hence the stability of gauged extended supergravity [5] and of supergravity coupled supersymmetrically to super-matter [25], even if the scalar potentials are unbounded below.

Acknowledgements. I would like to thank G W Gibbons and S. W Hawking for useful discussions. I would also particularly like to thank M Roček, a conversation with whom suggested many of the ideas developed here

Note Added in Proof. S. W. Hawking has recently shown that for a space-time to admit an asymptotic group of motions that is $0(3,2)$, it is necessary that the gravitational field satisfy one of the boundary conditions given by Breitenlohner and Freedman [22] These boundary conditions are then necessary for the existence of asymptotic Killing vectors and spinors and should be used in the positive mass theorem for AAdS spaces

After completion of this paper, I received a paper by $S$ Deser giving an alternative discussion of Witten's proof in terms of classical supergravity

\section{References}

1 Trautman, A : Conservation laws in general relativity. In: Gravitation, L Witten (ed). New York: John Wiley \& Sons 1962 pp 169-198

2 Arnowitt, R., Deser, S. Misner, C W : Canonical variables for general relativity. Phys Rev. 117, 1595-1602 (1960); Energy and the criteria for radiation in general relativity. Phys Rev 118, 11001104 (1960); Co-ordinate invariance and energy expressions in general relativity. Phys. Rev. 122, 997-1006 (i961)

3 Bondi, H., van der Burg, M. G. J, Metzner, A. W. K.: Gravitational waves in general relativity VII. Waves from axi-symmetric isolated systems. Proc R Soc. (London) A269, 21-52 (1962)

4. Abbott, F. L, Deser, S.: Stability of gravity with a cosmological constant. Nucl Phys. B195, 76-96 (1982)

5 Gibbons, G. W., Hull, C. M., Warner, N. P.: The stability of gauged supergravity. D.A.M.T.P. Preprint (1982)

6 Schoen, R., Yau, S.-T.: On the proof of the positive mass conjecture in general relativity. Commun. Math Phys 65, 45-76 (1979), Proof of the positive mass theorem II Commun. Math Phys. 79, 231-260 (1982)

7 Schoen, R, Yau, S -T, Proof that the Bondi mass is positive. Phys. Rev. Lett 48, 369-371 (1982); Horowitz, G W, Perry, M J : Gravitational energy cannot become negative. Phys Rev Lett 48 , 371-374 (1982)

8. Gibbons, G. W., Hawking, S W., Horowitz, G. W., Perry, M. J.: Positive mass theorems for black holes Commun. Math Phys 88, 295-308 (1983)

9. Gross, D. J., Perry, M. J., Yaffe, L G.: Instability of flat space at finite temperature. Phys. Rev. D25, 330-335 (1982)

10. Witten, E.: Instability of the Kaluza-Klein vacuum. Nucl Phys. B195, 481-492 (1982)

11. Witten, E.: A New proof of the positive energy theorem. Commun. Math. Phys. 80, 381-402 (1981)

12. Deser, S., Teitelboim, C.: Supergravity has positive energy Phys. Rev. Lett. 39, 249-252 (1977)

13. Grisaru, M. T.: Positivity of the energy in Einstein theory Phys Lett. 73B, 207-208 (1978)

14 Teitelboim, C : Surface integrals as symmetry generators in supergravity theory Phys. Lett. 69B, 240-244 (1977)

15 Nester, J. M.: A new gravitational energy expression with a simple positivity proof Phys Lett. 83A, 241-242 (1981)

16. Israel, W., Nester, J. M.: Positivity of the Bondi gravitational mass. Phys. Lett. 85A, 259-260 (1981) 
17 Choquet-Bruhat, Y., Christodoulou, D.: Elliptic Systems in $H_{s, s}$ spaces on manifolds which are Euclidean at infinity Acta. Math 146, 124-150 (1981); Parker, T, Taubes, C H : On Witten's proof of the positive energy theorem. Commun. Math. Phys. 84, 223-238 (1982); Reula, O : Existence theorem for solutions of Witten's equation and non-negativity of total mass $\mathbf{J}$ Math Phys 23, 810-814 (1982)

18 Freedman, D. Z, van Nieuwenhuizen, P, Ferrara, S.: Progress towards a theory of supergravity. Phys Rev D13, 3214-3218 (1976); Deser, S., Zumino, B : Consistent supergravity Phys Lett. 62B, 335-337 (1976); Freedman, D Z., van Nieuwenhuizen, P : Properties of supergravity theory Phys Rev D14, 912-916 (1976)

19. Townsend, P. K.: Cosmological constant in supergravity Phys Rev D15, 2802-2804 (1977)

20 Cremmer, E., Scherk, J : Algebraic simplifications in supergravity theories Nucl. Phys B127, 259268 (1977)

21 Dirac, P. A. M.: Generalized Hamiltonian dynamics Can J Math. 2, 129-148 (1950)

22 Breitenlohner, P., Freedman, D. Z.: Positive energy in anti-de Sitter backgrounds and gauged extended supergravity Phys Lett 115B, 197-201 (1982); Stability in gauged extended supergravity M I T Preprint (1982)

23 Arnowitt, R, Deser, S, Misner, C W : The dynamics of general relativity in Gravitation, Witten, L (ed ) New York: John Wiley \& Sons pp 227-265 1962; Deser, S., Isham, C. J.: Canonical Vierbein form of general relativity Phys. Rev D14, 2505-2510 (1976); Nelson, J. E., Teitelboim, C: Hamiltonian for the Einstein-Dirac field Phys. Lett 69B, 81-85(1977); Deser, S., Kay, J. H., Stelle, K S : Hamiltonian formulation of supergravity. Phys. Rev. D16, 2448-2455 (1977); Pilati, M.: The canonical formulation of supergravity Nucl Phys B132, 138-154 (1978)

24 Horowitz, G T, Strominger, A : On Witten's expression for gravitational energy Princeton Preprint (1982)

25 Hull, C M : (in preparation)

26 Gibbons, G W, Hull, C M.: A Bogomolny bound for general relativity and solitons in $N=2$ supergravity Phys Lett 109B, 190-194 (1982)

Communicated by S Hawking

Received March 10, 1983; in revised form April 20, 1983 
